\title{
Rapid Detection of the Three Celiac Disease Risk Genotypes HLA-DQ2.2, HLA-DQ2.5, and HLA-DQ8 by Multiplex Ligation-Dependent Probe Amplification
}

\author{
Raymon Vijzelaar, Ellen van der Zwan, ${ }^{2}$ Adriaan van Gammeren, ${ }^{3}$ Rizkat Yilmaz, Alice Verheul, ${ }^{2}$ \\ Ingrid van Hoogstraten, ${ }^{4}$ Ellen de Baar, ${ }^{3}$ Lianne Schrauwen, ${ }^{3}$ and Wouter Kortlandt ${ }^{2}$
}

Background: Genotyping of HLA-DQ2.2, HLA-DQ2.5, and HLA-DQ8 is important in celiac disease (CD). The absence of these three genotypes has a strong negative predictive value. Methods: We designed multiplex ligation-dependent probe amplification (MLPA) for the combined detection of HLA-DQ2.2, HLA-DQ2.5, and HLA-DQ8. The MLPA probe mix was validated against a set of 59 samples characterized by conventional techniques. Results: The MLPA assay genotyped all 59 samples correctly when compared to the results obtained by PCR-SSCP/HD or PCR-SSO and PCR-SSP. Conclusion: The MLPA assay provides a reliable single-reaction analysis of the CD risk genotypes HLA-DQ2.2, HLA-DQ2.5, and HLA-DQ8 allowing for stratification or exclusion of disease risk.

\section{Introduction}

C ELIAC DISEASE (CD, \#212750; OMIM) is a chronic small-intestinal enteropathy, characterized by an autoimmune response triggered by gluten proteins found in wheat, barley, or rye resulting in small intestine mucosal injury. CD is associated with HLA-DQ2.5, HLA-DQ2.2, and HLA-DQ8 molecules of the human leukocyte antigens (HLA) class II (Mubarak et al., 2013; Barakauskas et al., 2014). The heterodimeric HLA-DQ protein is found on the surface of antigen-presenting cells and consists of the $\alpha$ and $\beta$ subunits, which are encoded by HLA-DQA1 and HLA$D Q B 1$ genes, respectively (Monsuur et al., 2008; Megiorni and Pizzuti, 2012).

More explicitly, HLA genotypes HLA-DQ2.5 (HLA$D Q A 1 * 05$ and $H L A-D Q B 1 * 02)$, HLA-DQ8 (HLA-DQA1*03 and $H L A-D Q B 1 * 03: 02$ ), and more recently, found by $\mathrm{Mu}-$ barak et al. (2013), HLA-DQ2.2 (HLA-DQA1*02 and HLA$D Q B 1 * 02$ ) have been highly associated with CD (Table 1 ). However, $\sim 30-40 \%$ of the healthy population carries one of these risk variants, while the worldwide prevalence of $\mathrm{CD}$ is about $1 \%$. Consequently, the presence of HLA-DQ risk alleles alone is not the only prerequisite to develop $\mathrm{CD}$.

The development of CD appears to be multifactorial, including potential, additional genetic, and environmental factors (Megiorni and Pizzuti, 2012; Ludvigsson et al., 2014). Genotyping of the $H L A-D Q A 1$ and $H L A-D Q B 1$ genes is important since genetic HLA-DQ2.2, HLA-DQ2.5, and HLA-DQ8 testing has a very strong negative predictive value (Anderson et al., 2013; Ludvigsson et al., 2014). Testing for relevant alleles is recommended for risk stratification of individuals genetically susceptible to $\mathrm{CD}$, such as in firstdegree relatives. It is also recommended in case of autoimmune conditions such as type I diabetes or thyroiditis and in specific genetic disorders such as Down, Turner, or Williams syndrome (Fasano and Catassi, 2012; Barakauskas et al., 2014). Hence, the development of a single assay that can detect all three genotypes is essential to identify or exclude people at risk for $\mathrm{CD}$.

Several studies show that nearly $100 \%$ of CD patients contain one or combination of HLA-DQ2.5, HLA-DQ2.2, or HLA-DQ8 (Megiorni et al., 2009; Mubarak et al., 2013; Lund et al., 2015). In 90\% of CD cases, the DQ2.5 haplotype is found. The remaining $10 \%$ of $\mathrm{CD}$ patients are positive for either DQ2.2 or DQ8 or are homozygous for $H L A$ $D Q B 1 * 02$ (Mubarak et al., 2013; Ludvigsson et al., 2014). The European Society for Paediatric Gastroenterology, Hepatology, and Nutrition guideline (ESPGHAN) (Husby et al., 2012) recommends genetic testing on the presence of only HLA-DQ2.5 and HLA-DQ8. However, Mubarak et al. (2013)

\footnotetext{
${ }^{1}$ MRC-Holland BV, Amsterdam, The Netherlands.

${ }^{2}$ Department of Clinical Chemistry, Hematology and Immunology, Diakonessenhuis, Utrecht, The Netherlands.

${ }^{3}$ Department of Clinical Chemistry and Hematology, Amphia Hospital, Breda, The Netherlands.

${ }^{4}$ Department of Pathology, VU University Medical Center, Amsterdam, The Netherlands.
}

(C) Raymon Vijzelaar et al. 2018; Published by Mary Ann Liebert, Inc. This Open Access article is distributed under the terms of the Creative Commons License (http://creativecommons.org/licenses/by/4.0), which permits unrestricted use, distribution, and reproduction in any medium, provided the original work is properly cited. 
Table 1. Probes for the Detection of HLA Alleles Associated with Celiac Disease

\begin{tabular}{|c|c|c|c|c|c|}
\hline Length & Partial sequence (24 nt adjacent to ligation site) & HLA allele & $D Q 2.5$ & $D Q 2.2$ & $D Q 8$ \\
\hline 200 & TAAATCCTTCTC-GGAGAGGTCTCA & DQA1*05 & + & - & - \\
\hline 332 & TGCTGAGGAGAG-TTATGACTGCAA & DQA $1 * 05$ & + & - & - \\
\hline 183 & AACTATGGGGTA-TGGGGACAAACA & DQB $1 * 02$ & + & + & - \\
\hline 319 & TATAACCGAGAA-GAGATCGTGCGC & $\mathrm{DQB} 1 * 02$ & + & + & - \\
\hline 221 & AGACTGTCTGGA-AGTTGCCTCTGT & DQA $1 * 02$ & - & + & - \\
\hline 288 & AACCCCAGGGCA-33 nt spanning oligo-GCTGGAATCTCA & DQA $1 * 02$ & - & + & - \\
\hline 177 & GGTCCCTCTGGG-CAGTACAGCCAT & DQA $1 * 03$ & - & - & + \\
\hline 142 & ATTTGCACTGAC-35 nt spanning oligo-TGATTAAACGCT & DQA $1 * 03$ & - & - & + \\
\hline 233 & GACCCGGGCGGA-32 nt spanning oligo-TGGAGCTCCGCA & $\mathrm{DQB} 1 * 03$ & - & - & + \\
\hline 135 & CAGTACTCGGCG-30 nt spanning oligo-TACACCCCCACG & DQB $1 * 03: 02 * 03: 05$ & - & - & + \\
\hline 148 & GGGCCGGGGCCT-40 nt spanning oligo-CCAGTTTAAGGG & DQB1*03:02 *03:03 & - & - & + \\
\hline
\end{tabular}

HLA, human leukocyte antigens.

showed that 9 of $155 \mathrm{CD}$ patients were negative for both HLA-DQ2.5 and HLA-DQ8, but positive for HLA-DQ2.2 demonstrating that HLA-DQ2.2 should be also considered as a susceptible HLA-DQ haplotype in CD, even without the presence of HLA-DQ2.5 or HLA-DQ8 (Mubarak et al., 2013). This was confirmed in a recent study by Lund et al. (2015).

Previously, we published a novel multiplex ligationdependent probe amplification (MLPA) assay to confirm or exclude HLA-DQ2.5 and HLA-DQ8 genotypes (Van Beek et al., 2013). This MLPA assay has been validated by genotyping 155 samples using single-strand conformation polymorphism and heteroduplex analysis (PCR-SSCP/HD) at the VU University Medical Center (Van Beek et al., 2013). We have improved our previously published MLPA assay by including probes for the simultaneous detection of the HLADQ2.2 genotype, in addition to HLA-DQ2.5 and HLA-DQ8. Results obtained by this redesigned MLPA assay were $100 \%$ consistent with the results of conventional methods such as PCR-SSCP/HD, PCR-SSO, and PCR-SSP.

\section{Materials and Methods}

The MLPA probemix P438-D1 (MRC-Holland, Amsterdam, The Netherlands) was developed to simultaneously detect the $\alpha$ and $\beta$ subunits of the heterodimeric DQ2.2, DQ2.5, and DQ8 molecules. For each allele, that is, HLA$D Q A 1 * 02, H L A-D Q A 1 * 03, H L A-D Q A 1 * 05$, and HLA$D Q B 1 * 02$, two probes have been added to the probemix (Table 1 and Fig. 1). In comparison to the previous version, two probes for $H L A-D Q A 1 * 05$ have been removed from the probemix. It was shown that two probes for each allele are sufficient for detection of the relevant allele. Therefore, the two best performing probes have been selected. Nine reference probes, targeting other chromosomes for internal quality control on the polymerase chain reaction (PCR) process, have also been incorporated. MLPA probe design requires the presence of unique sequences to prevent the amplification of homologous sequences (Schouten et al., 2002). The design of a single-MLPA probe unique for $H L A-$ $D Q B 1 * 03: 02$ (HLA-DQ8) detection is impossible due to the large number of $H L A-D Q B 1$ gene variants. Therefore, a combination of three probes has been included to detect the $H L A-D Q B 1 * 03: 02$ allele of the HLA-DQ8 $\beta$-unit.

The MLPA assay was performed according to manufacturers' instructions (www.mlpa.com). In brief, the MLPA reaction requires $50-100 \mathrm{ng}$ of sample DNA, which is denatured and mixed with the MLPA probemix before an overnight hybridization at $60^{\circ} \mathrm{C}$. After the ligation $\left(15^{\prime}\right.$ at $54^{\circ} \mathrm{C}$ ) and amplification by PCR (35 cycles), the PCR fragments are analyzed on a capillary electrophoresis. Electrophoresis was carried out on an ABI 3130 sequencer (Life Technologies/Applied Biosystems, Carlsbad, CA) by the Amphia Hospital (Breda, The Netherlands) or a Beckman 8800 sequencer (Beckman Coulter, Woerden, The Netherlands) by the Diakonessenhuis Hospital (Utrecht, The Netherlands).

Reference genotyping was performed by either the VU University Medical Center (Amsterdam, The Netherlands) using a PCR-SSCP/HD-based method or Sanquin (Amsterdam, The Netherlands) using the sequence-specific oligonucleotideprimed PCR (PCR-SSO) and sequence-specific primerbased PCR (PCR-SSP) (Barakauskas et al., 2014). The Diakonessenhuis Hospital evaluated 35 samples and compared results to those obtained by the VU University Medical Center. The Amphia Hospital evaluated 24 samples and compared their results to those of Sanquin. The samples have been selected with a bias for HLA-DQ2.2.

\section{Results and Discussion}

The MLPA probemix P438-D1 has been validated by two centers, the Diakonessenhuis Hospital and Amphia Hospital, using 59 samples consisting of different HLA-DQ variants. The sample composition consisted of 19 HLA-DQ2.2 positive (Fig. 1B), 3 HLA-DQ2.5 positive, 6 HLA-DQ8 positive, 4 both HLA-DQ2.2 and HLA-DQ8 positive (Fig. 1C), 11 both HLA-DQ2.2 and HLA-DQ2.5 positive (Fig. 1D), 3 both HLA-DQ2.5 and HLA-DQ8 positive, and 13 HLA DQ2.5, DQ2.2, or DQ8 negative (Fig. 1A). Results obtained by the MLPA assay were $100 \%$ consistent with the reference genotyping results (Table 2 ).

Current guidelines (ESPGHAN) suggest that $\mathrm{CD}$ can be excluded in DQ2.5- and DQ8-negative patients (Husby et al., 2012; Mubarak et al., 2013). Recently, it has been demonstrated that DQ2.2, with a prevalence of about 5\%, which is as frequent as DQ8, is another risk genotype for CD (Mubarak et al., 2013). Collectively, these three risk genotypes are found in almost all CD patients. We have included $H L A$ $D Q A 1 * 02$ allele-specific MLPA probes in this MLPA assay to detect the DQ2.2 genotype. 
A

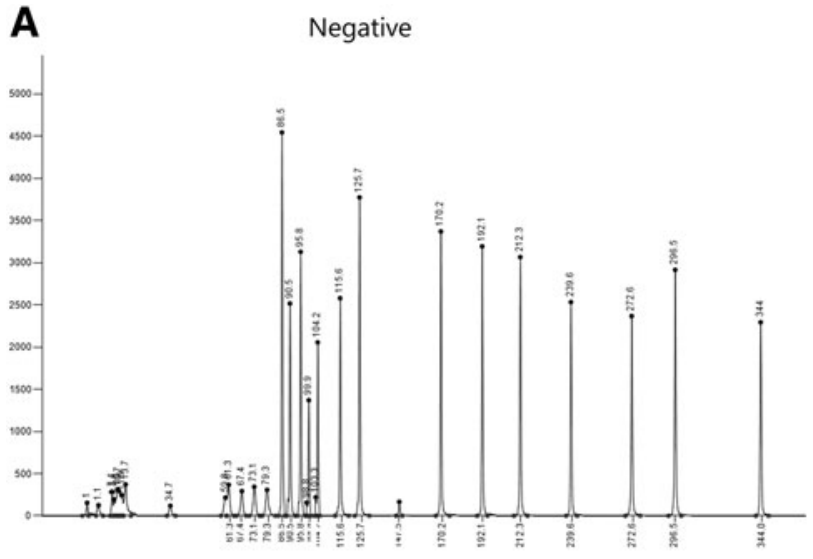

C

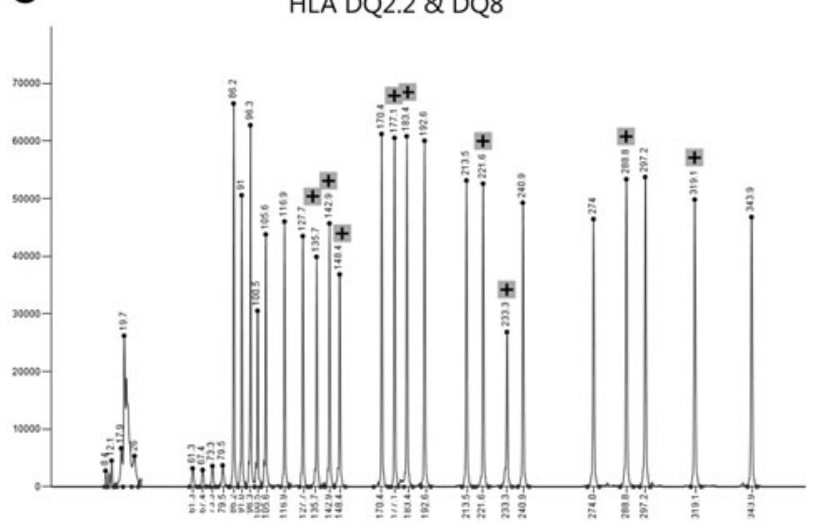

B HLA DQ2.2
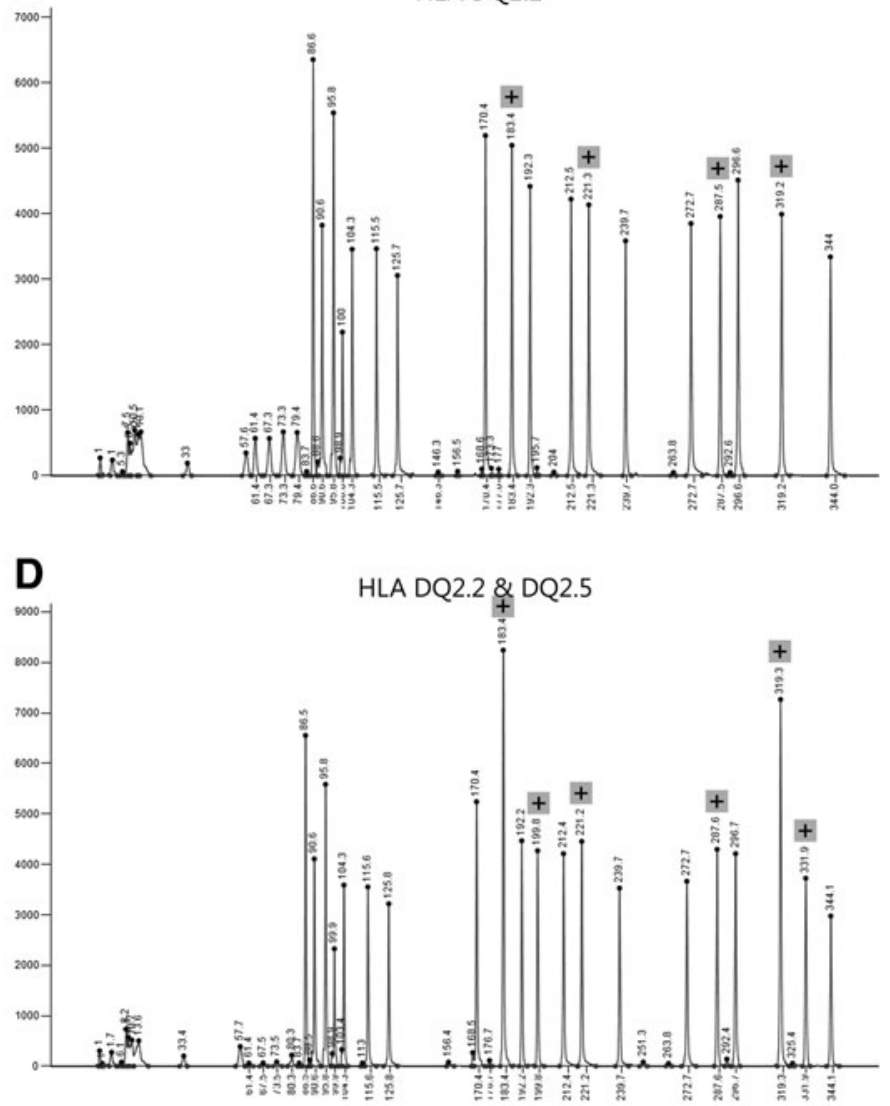

FIG. 1. Electropherograms of samples harboring different human leukocyte antigen (HLA) alleles detected in this study. (A) Sample negative for HLA-DQ2.2, HLA-DQ2.5, and HLA-DQ8. (B) HLA-DQ2.2-positive sample, showing a heterozygous signal for the HLA-DQB1*02 allele. (C) HLA-DQ2.2- and HLA-DQ8-positive sample. (D) HLA-DQ2.2- and HLADQ2.5-positive sample, showing a homozygous signal for the HLA-DQB1*02 allele. Probe identities are described in Table 1 by matching the corresponding length.

Validation of this renewed method by two independent institutes showed that the method with specific probes for all relevant $\mathrm{CD}$ risk genotypes is a very reliable and complete assay to exclude or stratify the risk for CD (Van Beek et al., 2013; Barakauskas et al., 2014). In addition, MLPA has been shown to be able to distinguish between heterozygous and homozygous state of the alleles (Van Beek et al., 2013; Barakauskas et al., 2014), which is of importance since ho- mozygous $H L A-D Q B 1 * 02$ in the presence or absence of $H L A-D Q A 1 * 02$ or HLA-DQA1*05 also predisposes for CD (Megiorni et al., 2009).

In conclusion, the new MLPA probemix is able to correctly detect multiple targets within one reaction, in contrast to other more conventional multistep technologies, and provides a quick, reliable, and complete method to determine all three HLA-DQ genotypes relevant for CD.

Table 2. Genotyping Results of Samples Rested with the Improved MLPA Assay (P438-D1) COMPARED WITH ReFERENCE Methods

\begin{tabular}{lcccc}
\hline Genotype combination & $\begin{array}{c}\text { Reference method 1: } \\
\text { PCR-SSO/SSP }\end{array}$ & $\begin{array}{c}\text { Reference method 2: } \\
\text { PCR-SSCP/HD }\end{array}$ & Results by MLPA & Specificity \\
\hline HLA-DQ2.2, -DQX & 4 & 15 & 19 & 11 \\
HLA-DQ2.2, -DQ2.5 & 4 & 7 & 4 & $11 / 11$ \\
HLA-DQ2.2, -DQ8 & 0 & 4 & 3 & $4 / 4$ \\
HLA-DQ2.5, -DQX & 0 & 3 & 3 & $3 / 3$ \\
HLA-DQ2.5, -DQ8 & 3 & 3 & 6 & $3 / 3$ \\
HLA-DQ8, -DQX & 3 & 3 & 13 & $6 / 6$ \\
HLA-DQX, -DQX & 10 & 35 & 59 & $5 / 13$ \\
Total & 24 & $39 / 59$ \\
\hline
\end{tabular}

DQX, any HLA type other than HLA-DQ2.2, HLA-DQ2.5, and HLA-DQ8; MLPA, multiplex ligation-dependent probe amplification; SSCP/ $\mathrm{HD}$, single-strand conformation polymorphism/heteroduplex; SSO/SSP, sequence-specific oligonucleotide/sequence-specific primer based. 


\section{Acknowledgment}

The authors thank their colleagues at MRC-Holland for useful discussions and the supply of MLPA kits and reagents.

\section{Author Disclosure Statement}

R.V. and R.Y. are employed by MRC-Holland, provider of commercially available MLPA assays. All other authors have no conflict of interests.

\section{References}

Anderson RP, Henry MJ, Taylor R, et al. (2013) A novel serogenetic approach determines the community prevalence of celiac disease and informs improved diagnostic pathways. BMC Med 11:188.

Barakauskas VE, Lam GY, Estey MP (2014) Digesting all the options: laboratory testing for celiac disease. Crit Rev Clin Lab Sci 51:358-378.

Fasano A, Catassi C (2012) Clinical practice. Celiac disease. N Engl J Med 367:2419-2426.

Husby S, Koletzko S, Korponay-Szabó IR, et al. (2012) European Society for Pediatric Gastroenterology, Hepatology, and Nutrition guidelines for the diagnosis of coeliac disease. J Pediatr Gastroenterol Nutr 54:136-160.

Ludvigsson JF, Bai JC, Biagi F, et al. (2014) Diagnosis and management of adult coeliac disease: guidelines from the British Society of Gastroenterology. Gut 63:1210-1228.

Lund F, Hermansen MN, Pederson MF, et al. (2015) Mapping of HLA-DQ haplotypes in a group of Danish patients with celiac disease. Scand J Clin Lab Invest 75:519-522.
Megiorni F, Mora B, Bonamico M, et al. (2009) HLA-DQ and risk gradient for celiac disease. Hum Immunol 70:55-59.

Megiorni F, Pizzuti A (2012) HLA-DQA1 and HLA-DQB1 in Celiac disease predisposition: practical implications of the HLA molecular typing. J Biomed Sci 19:88.

Monsuur AJ, de Bakker PI, Zhernakova A, et al. (2008) Effective detection of human leukocyte antigen risk alleles in celiac disease using tag single nucleotide polymorphisms. PLoS One 3:e2270.

Mubarak A, Spierings E, Wolters VM, et al. (2013) Human leukocyte antigen DQ2.2 and celiac disease. J Pediatr Gastroenterol Nutr 56:428-430.

Schouten JP, McElgunn CJ, Waaijer R, et al. (2002) Relative quantification of 40 nucleic acid sequences by multiplex ligationdependent probe amplification. Nucleic Acids Res 30:e57.

Van Beek EM, Roelandse-Koop EA, Vijzelaar R, et al. (2013) A multiplex assay to rapidly exclude HLA-DQ2.5 and HLADQ8 expression in patients at risk for celiac disease. Clin Chem Lab Med 51:1191-1198.

Address correspondence to: Wouter Kortlandt, PhD Department of Clinical Chemistry, Hematology and Immunology Diakonessenhuis Bosboomstraat 1 3582 KE Utrecht The Netherlands

E-mail: wkortlandt@diakhuis.nl 